Canadian Journal of Regional Science

Revue canadienne des sciences régionales

\title{
La professionnalisation comme outil de protection du territoire agricole québécois : "Il faut empêcher que se constituent des petites fermes non rentables "
}

\section{Julie Francoeur et Élisabeth Abergel}

Volume 43, numéro 1, 2020

URI : https://id.erudit.org/iderudit/1083578ar

DOI : https://doi.org/10.7202/1083578ar

Aller au sommaire du numéro

\section{Éditeur(s)}

Canadian Regional Science Association / Association canadienne des sciences régionales

\section{ISSN}

0705-4580 (imprimé)

1925-2218 (numérique)

Découvrir la revue

\section{Citer cet article}

Francoeur, J. \& Abergel, É. (2020). La professionnalisation comme outil de protection du territoire agricole québécois : « Il faut empêcher que se constituent des petites fermes non rentables ". Canadian Journal of Regional Science / Revue canadienne des sciences régionales, 43(1), 16-27.

https://doi.org/10.7202/1083578ar
Résumé de l'article

Cet article s'intéresse au rôle du zonage agricole dans la professionnalisation de l'agriculture québécoise et dans la limitation de l'accès à la profession d'agriculteur (1), ainsi qu'à ses conséquences pour le développement et le renouvellement de l'agriculture aujourd'hui (2). À partir d'anciens débats sur la protection du territoire et des activités agricoles, nous montrons que la question de la protection du territoire agricole est indissociable de celle de la professionnalisation de l'agriculture, et que les évolutions actuelles concernant la multifonctionnalité, bien qu'ambigües, invitent à s'interroger tant sur le modèle québécois en matière de protection des terres agricoles que sur les critères exclusifs sur lesquels repose la professionnalité agricole. 


\section{CANADIAN JOURNAL OF REGIONAL SCIENCE REVUE: CANADIENNE DES SCIENCES RÉGIONALES}

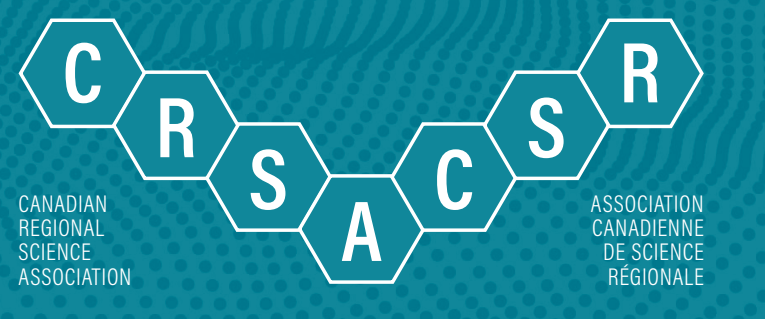

LA PROFESSIONNALISATION COMME OUTIL DE PROTECTION DU TERRITOIRE AGRICOLE QUÉBÉCOIS: "IL FAUT EMPÊCHER QUE SE CONSTITUENT DES PETITES FERMES NON RENTABLES ${ }^{1}$ "

\section{Julie Francoeur, Élisabeth Abergel}

Julie Francoeur, Ph.D.

Montréal, Québec, Canada

francoeur.julie@uqam.ca

\author{
Élisabeth Abergel, professeure \\ Département de sociologie, Université du Québec à Montréal \\ Institut des sciences de l'environnement \\ 400, rue Sainte-Catherine Est, A-5325 \\ Montréal, Québec, Canada \\ abergel.elisabeth@uqam.ca
}

Résumé: Cet article s'intéresse au rôle du zonage agricole dans la professionnalisation de l'agriculture québécoise et dans la limitation de l'accès à la profession d'agriculteur (1), ainsi qu'à ses conséquences pour le développement et le renouvellement de l'agriculture aujourd'hui (2). À partir d'anciens débats sur la protection du territoire et des activités agricoles, nous montrons que la question de la protection du territoire agricole est indissociable de celle de la professionnalisation de l'agriculture, et que les évolutions actuelles concernant la multifonctionnalité, bien qu'ambigües, invitent à s'interroger tant sur le modèle québécois en matière de protection des terres agricoles que sur les critères exclusifs sur lesquels repose la professionnalité agricole.

This paper analyzes the role played by agricultural zoning in both the professionalization of Quebec agriculture and in restricting access to the profession (1) including its impacts on the development and renewal of agriculture today (2). Based on historical debates about the protection of rural territory and agricultural activities, we demonstrate that the issue of territorial protection is intrinsically linked to the professionalization of agriculture. In addition, recent developments concerning multifunctionality, while remaining ambiguous, raise questions about Quebec's model of rural land protection and about the exclusion criteria which define the agricultural profession.

Mots-clés: Agriculture | espace rural | professionnalisation | zonage agricole | repaysanisation | Québec 
Au Canada, la protection des espaces agricoles constitue un objectif important de politique publique dans de nombreuses juridictions au niveau de l'État provincial, y compris au Québec. En particulier, la délimitation restrictive du domaine agricole, plus communément appelé le zonage agricole, représente le principal instrument de la législation québécoise en faveur de la protection des terres et des activités agricoles, en zones périurbaines et rurales.

S'inspirant du modèle en vigueur en Colombie-Britannique depuis 1973 (Pierce and Furuseth 1982; Hanna 1997; Stobbe, Eagle, and Kooten 2008; Stobbe et al. 2011; Newman, Powell, and Wittman 2015), le modèle québécois repose sur l'adoption, en 1978, de la Loi sur la protection du territoire agricole (LPTA), renommée Loi sur la protection du territoire et des activités agricoles (LPTAA) en vertu de la révision de 1996. Cette loi définit et protège aujourd'hui une zone agricole d'une superficie de plus de 63500 kilomètres carrés, s'étendant sur le territoire de 955 municipalités (Commission de protection du territoire agricole 2018), ce qui représente moins de $2 \%$ de la superficie totale de la province.

Différents facteurs ont contribué à justifier cette mesure qui, à son origine, était contestée non dans son principe même, mais dans ses modalités (Assemblée nationale du Québec 1978a). Un premier argument déplorait le manque de planification en matière d'aménagement eu égard à l'urbanisation accélérée des terres agricoles dans la grande région de Montréal (Vachon 2011). Une étude produite par le ministère des Affaires municipales au cours de l'année précédant l'adoption de la loi estimait la consommation d'espaces agricoles par l'urbanisation dans cette seule région à 20000 hectares entre 1964 et 1975, soit l'équivalent de plus de 200 fois les Plaines d'Abraham (Québec). Accueillant des populations issues de l'exode rural et de l'immigration, une expansion urbaine et périurbaine non maitrisée compromettait le développement agricole local et la pérennité de l'agriculture urbaine et périurbaine, où l'on retrouvait, disait-on, la majorité des maraichers (Assemblée nationale du Québec 1978a).

Un second argument présentait la loi à adopter comme la dimension foncière d'une politique agricole nationaliste (Debailleul 1988). Suivant l'adoption de la loi, la publication du document «Bâtir le Québec » en 1979 et la diffusion de la série «Nourrir le Québec» de 1980 à 1985, de même que les rapports de la Conférence socio-économique sur l'agro-alimentaire déposés en 1978, 1979 et 1980, devaient témoigner de ce projet de société indépendantiste porté par le gouvernement au pouvoir.

Dans cet article, nous invitons le lecteur et la lectrice à poser un regard sur une autre dimension peu étudiée de la politique de zonage agricole au Québec en montrant l'importance que celle-ci devait revêtir dans l'imposition du modèle professionnel agricole québécois dans les années soixante-dix et, plus précisément, dans la limitation de l'accès à la profession d'agriculteur. Reprenant l'argument de Galeski (1967) concernant rôle des législations d'État interdisant de morceler les terres agricoles dans la professionnalisation de l'agriculture - entendue comme le processus de construction à la fois politique, idéologique et sociale du groupe des «agriculteurs professionnels» par l'exclusion de certaines catégories d'exploitants définis sur la base de leur altérité (petites surfaces, pluriactifs, produisant trop peu de ventes, etc.) (Rémy 1987) -, nous développons cette thèse en situant la mesure législative dans la continuité des initiatives destinées à assurer la parité des revenus aux producteurs agricoles (Commission Héon 1955; Commission royale d'enquête sur l'agriculture au Québec 1967; Commission de planification de l'agriculture canadienne 1969), à leur reconnaitre un statut professionnel (Loi sur les producteurs agricoles, 1972) et à protéger leurs pratiques; bref, dans la continuité de l'objectif global de la profession et du gouvernement de l'époque, qui est de professionnaliser l'agriculture. La recherche présentée repose sur une étude qualitative que nous avons conduite à partir d'un corpus principalement formé des verbatim des travaux parlementaires autour des lois 90 (LPTA) et 23 (LPTAA).

Blanchemanche et al. (2000) ont montré que, de façon générale, la pratique de l'agriculture emprunte de moins en moins aux formes d'emploi monoactif «à temps plein » valorisées et reconnues par l'environnement professionnel agricole, et que cette situation relève spécifiquement de la reconnaissance récente de la multifonctionnalité de l'agriculture dans les pays; la reconnaissance des activités agricoles produisant peu, voire très peu de ventes, mais participant au maintien du tissu social rural en représenterait selon elles un second aspect. Nous avançons que notre réflexion trouve son importance et sa pertinence dans ce contexte de légitimité nouvelle de la diversité des systèmes d'activité - porteuse de nouvelles formes de professionnalité à construire, également mise en lumière par Jan Douwe van der Ploeg (2012) avec le concept de «repaysanisation » de l'agriculture et par Christian Mouchet et Jean-François Le Clanche (2016) dans leurs récents travaux sur les petites exploitations françaises.

Au Québec, en effet, les premiers changements concernant la reconnaissance politique de la multifonctionnalité de l'agriculture impliquent une reconnaissance des différents facteurs qui freinent le développement de nouvelles formes d'agriculture dans la province. En particulier, la LPTAA est remise en question pour contraindre l'installation ou le développement de projets agricoles sur petites surfaces (Ouimet 2009). À ce titre, on admet de plus en plus que le renforcement de la protection du territoire agricole dans les années soixante-dix a été suivi d'un mouvement de concentration des exploitations (Proulx and Sauvé 2007), qui ne cesse aujourd'hui de prendre de l'ampleur. À ce sujet, le dernier Recensement de l'agriculture a montré que la superficie moyenne des entreprises agricoles québécoises s'est stabilisée autour de 113 hectares entre 2011 et 2016 (Gouvernement du Canada, Statistique Canada 2016), alors qu'elle était de 78 hectares au moment du Recensement précédant l'adoption de la loi, reflétant le caractère socio-spatial de la réorganisation en cours.

Jusqu'à récemment, les effets des entreprises agricoles de petite taille sur le secteur agricole étaient considérés négativement, pour ralentir le rythme d'expansion des entreprises professionnelles les avoisinant (Assemblée nationale du Québec 1978j; Schmitt and Coujard 1985). En particulier, celles situées dans leur aire d'expansion étaient réputées faire obstacle à leur évolution, pourtant facilitée par la loi et l'accès au crédit. Aujourd'hui, ce sentiment de menace semble vouloir faire place à une certaine reconnaissance de la contribution des entreprises agricoles de petite taille à l'atteinte d'objectifs sociaux et environnementaux relatifs à l'occupation dynamique du territoire (Saint-Pierre 2009).

Sur la base de la recherche présentée, nous montrons que la question de la protection du territoire agricole est indissociable de celle de la professionnalisation de l'agriculture, et que les évolutions actuelles, bien qu'ambigües (Francoeur 2018), invitent tant à s'interroger sur le modèle québécois en matière de protection des terres agricoles (fonctionnalisation et homogénéisation de l'espace rural, interdiction du morcellement, obligation de volume, pratique monoactive «à temps plein» de l'agriculture) que sur les critères exclusifs sur lesquels repose la professionnalité agricole - lesquels ont des conséquences directes pour le développement et le renouvellement de l'agriculture aujourd'hui.

\section{L'AGRICULTURE AU QUÉBEC, DES ENJEUX DE TAILLE}

L'agriculture québécoise a suivi, depuis la fin de la Seconde guerre mondiale, une évolution similaire à celle expérimentée par la majorité des pays industrialisés. Au cours de la décennie 1950, le soutien des revenus agricoles y devenait une préoccupation d'ordre public. 
L'intervention de l'État québécois devait alors assurer la parité agricole et la productivité de l'agriculture (Commission de planification de l'agriculture canadienne 1969), assumant que le développement de l'agriculture ne pouvait être assuré que par « des hommes conscients d'avoir atteint une certaine parité économique, sociale et politique » (Malassis 1979, 9).

La nomination de la commission Héon par le gouvernement de Maurice Duplessis en 1951 marqua en ce sens le début d'un vaste mouvement de modernisation de l'agriculture québécoise. L'agriculture devant répondre «aux besoins des populations urbaines » et assurer «aux travailleurs du sol une subsistance convenable et la possibilité d'y établir leur fils» (Commission Héon 1955), c'est au nom du bien commun (la mise sur le marché ordonnée des produits de la ferme) et des intérêts bien compris de la classe agricole (la parité sociale) que le rapport Héon exigea du «petit» producteur qu'il participe de ce mouvement ou qu'il se retire du marché. «En termes quantitatifs, les 40000 spécialistes, porteurs de l'avenir, demandent l'adaptation ou la disparition des 100000 exploitations qui ne participent pas au mouvement de modernisation marchande » (Morisset and Couture 2010, 17).

«Si nous cherchons la stabilité de l'agriculture, il faut lui assurer des revenus suffisants et non seulement comparables à ceux des ouvriers des villes, mais pour le moins équivalents à ceux des industriels dont la mise de capitaux d'exploitation est correspondante» (Commission Héon 1955, 261).

L'image alors renvoyée au «petit» producteur fut celle d'un être inutile, car en surnombre; pis: nuisible: «le mauvais producteur, comme le mauvais soldat, sert mal son pays et ses concitoyens » (Commission Héon 1955, 141). On dit de lui qu'il causait préjudices aux «bons » producteurs en leur faisant concurrence déloyale sur le marché organisé; de son indifférence ou de son hostilité agissante, qu'elles étaient susceptibles de réduire à néant les efforts déployés par ceux-ci.

«Le nombre très considérable de petits producteurs dont les produits sont de quantités minimes et de qualité incertaine est un facteur de mise-en-marché désordonnée et d'avilissement des prix, qui pénalise nos producteurs spécialisés » (Commission Héon 1955, 141-42).

Conscient qu'il aurait été malaisé pour une société démocratique de policer ses producteurs indésirables, le rapport Héon misa sur des efforts d'éducation et de vulgarisation visant à rendre «bon» le producteur «médiocre». Aussi recommanda-t-il à l'État de poursuivre les efforts de professionnalisation de l'agriculture en soutenant, favorisant et intensifiant l'enseignement agricole primaire, moyen et supérieur.

Le rapport Héon tabla au demeurant sur une formule plus impérative que l'éducation, et en cela peut-être moins insidieuse, en recommandant l'inspection et la classification obligatoires des produits de la ferme, ainsi que la création d'un office des marchés. Par suite du dépôt du rapport, l'Office des marchés agricoles fut créée et la pratique des conventions collectives, légalisée, en vertu de la loi de mise en marché. D'abord réduite, la portée de la loi s'étendit en 1965. Le principe des plans conjoints à extension juridique, prenant parfois la forme de «quotas», obligea alors la totalité des producteurs et des acheteurs à respecter les termes d'une convention, dès lors qu'une entente était conclue. Cette évolution des structures de mise en marché alla de pair avec un mouvement de regroupement autour de syndicats et de fédérations spécialisés. Dans la presse professionnelle et syndicale agricole (La Terre de chez nous), l'avenir de la profession fut alors étroitement lié à celui de l'organisation professionnelle.

«Seule une classe agricole organisée assurera l'avenir de l'agriculture » (Lemoine 1957, 5).
Malgré l'ensemble de ces mesures, les surplus agricoles (résultant des hauts niveaux de productivité) continuèrent de s'imposer comme un obstacle majeur à la parité entre les secteurs industriel et agricole. La parité fut finalement espérée d'un transfert massif de la population agricole vers les autres secteurs de l'économie. Instituée en 1965 «pour faire enquête sur les moyens à prendre pour que la production agricole rapport aux agriculteurs un revenu équilibré par rapport à celui des autres classes de la société » (Commission royale d'enquête sur l'agriculture au Québec 1967, 5), la Commission royale d'enquête sur l'agriculture au Québec, présidée par Nolasque April, demanda à ce que la diminution de la population agricole se poursuivre tant et aussi longtemps que la disparité soit ramenée à un niveau acceptable (Commission royale d'enquête sur l'agriculture au Québec 1967)

Nommée en 1967 dans un contexte de production excédentaire et de revenus à la baisse, la Commission de planification de l'agriculture canadienne aborda directement la question du revenu et déposa en 1969 un rapport intitulé «L'agriculture canadienne des années 1970 ». Ce rapport fit état «d'un mécontement généralisé attribuable à des revenus agricoles faibles, à des marchés et des prix incertains, à la surproduction, à de petites exploitations agricoles non viables et à des marchés d'exportation à la baisse». On y recommanda de réduire la population vivant de l'agriculture et d'inciter les jeunes agriculteurs à faible revenu à renoncer à l'agriculture. Au surplus, le rapport souligna l'importance, au nom de l'intérêt national comme celui des producteurs agricoles, de stabiliser la production, les prix et les revenus, afin d'accélérer le processus de sélection des exploitations les plus performantes au sein du monde agricole (Commission de planification de l'agriculture canadienne 1969).

La stabilisation des revenus et de la production devint dès lors un enjeu majeur de la politique agricole des États canadien et québécois. À partir de 1975, une loi canadienne vint garantir un prix de soutien pour certains produits, dont la liste sera revue subséquemment. Parallèlement, au Québec, un programme de stabilisation des prix et des revenus agricoles fut adopté, via la loi sur l'Assurance stabilisation des revenus agricoles (ASRA). Tout en adhérant au principe de parité des revenus, I'ASRA chercha à garantir un revenu minimum annuel à l'agriculteur «efficace» (Lavoie 1979). Dans les faits, il s'agissait moins de garantir à un groupe d'agriculteurs des revenus stables et suffisamment élevés, que d'outiller le développement de l'agriculture. En faisant reposer le calcul des cotisations et des compensations sur le concept de «cout de production» et en fixant celui-ci à partir des couts courants de production d'un modèle de ferme-type élaboré au ministère de l'Agriculture en collaboration avec la profession agricole, les législateurs permirent la sélection des producteurs les plus efficaces, ainsi que le transfert du surtravail (Lavoie 1979).

Entretemps, la création d'un statut de producteur agricole professionnel chercha à assurer à un groupe restreint de producteurs le contrôle de l'exercice du métier d'agriculteur. Adopté le 31 mai 1972, le projet de loi 64, déposé en 1971 par le ministre Toupin, confia à la Régie des marchés agricoles et alimentaires (RMAAQ), déjà responsable de la Loi sur la mise en marché des produits agricoles, alimentaires et de la pêche, la fonction d'assurer la protection du statut de producteur agricole. On lui donna, par force de loi, la compétence à déclarer si une personne était, ou n'était pas, productrice agricole. Seules les personnes réalisant minimalement $3000 \$$ de recettes brutes avec la vente de leurs produits agricoles furent alors retenues. Il fut établi que ce seuil devait être révisé périodiquement afin de refléter les futurs développements de la profession. Aujourd'hui établi à 5000 \$, il reste débattu au sein du secteur. Certaines estimations indiquent que 7000 à 10000 entreprises agricoles suffiraient à l'ensemble de la production agricole québécoise (Bouchard 2014). Michel Morisset, titulaire au Département d'agroéconomie de I'Université Laval nominé à titre de conseiller expert du ministère de 
I'Agriculture, des Pêcheries et de l'Alimentation du Québec (MAPAQ) à l'occasion des consultations en vue de l'élaboration de la politique agricole de 2013, est de cet avis. Dans un article paru dans le quotidien Le Soleil en 2012, Morisset (2012) dénonçait «le développement d'une classe d'entrepreneurs fiscaux » responsable de nuire à la légitimité future de la profession agricole.

« Il y a peu ou pas de profession ou de métier où l'accès comporte si peu de contraintes que l'agriculture. Qu'est-ce que vendre pour $5000 \$$ de produits alors que la majorité des professions exigent une formation collégiale ou universitaire, la participation à un ordre professionnel, ou encore l'acquisition d'un certificat de compétence à la suite d'une période d'apprentissage» (Morisset 2012)?

Répondant à cette intervention, Benoit Girouard, alors président de I'Union paysanne, rétorquait le lendemain que «l'avenir appartient aux petites et moyennes entreprises agricoles, et qu'il passe par l'accroissement du nombre de fermes à dimension humaine » (Girouard 2012).

Instituée quelques années plus tard, en 1978, la CPTAQ allait contribuer à restreindre encore davantage l'accès au statut de producteur agricole au motif de protéger les sols jugés de bonne qualité pour l'agriculture, et donc zonés agricoles en vertu de la LPTA nouvellement adoptée. Dès lors, seuls les individus ayant l'agriculture pour activité principale allaient être autorisés à acquérir un lot (Organisation de coopération et de développement économiques 2010).

\section{LE MODÈLE D'AGRICULTURE PROFESSIONNELLE PRÉCONISÉ PAR LA CPTAQ}

À partir de 1978, la Loi sur la protection du territoire agricole (LPTA) allait désigner une partie du territoire québécois comme «région agricole». Issue du projet de loi 90, cette loi devait se baser sur l'in- ventaire des terres du Canada (ITC), établi en 1963 dans le cadre de la Loi sur l'aménagement rural et le développement agricole (ARDA) du gouvernement fédéral de John George Diefenbaker. Couvrant l'ensemble des provinces atlantiques, de même que les régions habitées du Québec, de l'Ontario et de I'Ouest canadien, cet inventaire répertoriait et divisait les terres canadiennes en sept classes selon leurs aptitudes ou leurs limitations respectives en matière de production agricole - des sols se prêtant aux cultures ordinaires à rendement continu aux sols ne se prêtant ni à l'agriculture ni à la culture permanente des herbages (Institut de recherche et de développement en agroenvironnement 2019).

Dans le projet initial déposé le 9 novembre 1978, l'identification des basses terres du Saint-Laurent et de l'Outaouais en tant que «régions agricoles désignées » fut justifiée par le système de classement des terres instauré par le gouvernement canadien (figure 1). Dans ces régions, certaines dispositions du projet de loi entrèrent en vigueur dès le dépôt du projet, notamment l'ensemble des interdictions en matière d'utilisation, de lotissement et d'aliénation des lots. L'idée était d'éviter «toute vague de spéculation» suivant «la publication inévitable du projet de loi » (Assemblée nationale du Québec 1978a).

À la demande insistante de la profession organisée (UPA) qui craignait certains dérapages nuisibles à l'extérieur de la région agricole désignée ${ }^{2}$, la loi fut finalement appliquée dans l'ensemble des régions préalablement exclues, à l'exception de l'île de Montréal où l'on retrouvait pourtant encore de nombreux maraichers. Dans l'Est-du-Québec, la loi consacra une vocation agricole exclusive à de nombreux rangs jugés non aptes à l'agriculture dans le cadre du Bureau d'aménagement de l'Est-du-Québec (BAEQ), quelques années plus tôt (Vachon 2011). Ailleurs, d'importantes superficies impropres à l'agriculture furent inclues, sous prétexte que les développements technologiques à venir les rendraient propices à l'agriculture (Vachon 2011).

Figure 1. Cartographie du zonage agricole au Québec

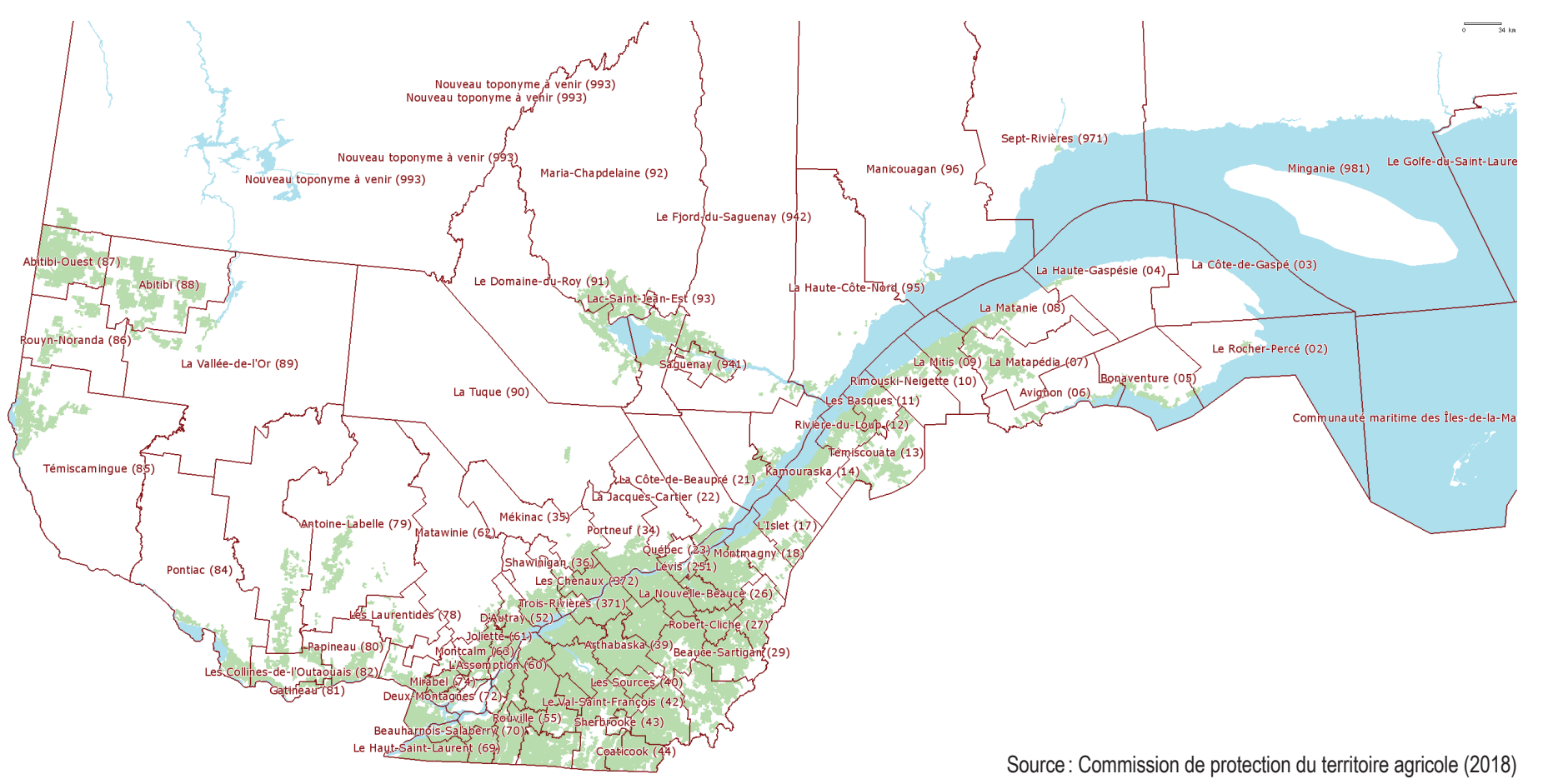

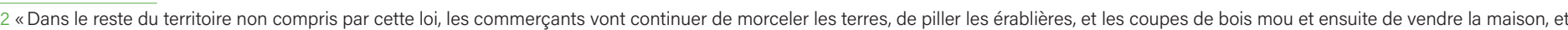

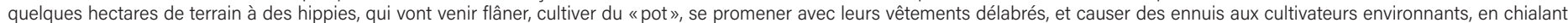
contre le bruit des machines agricoles et la senteur du fumier » (Fortier 1978). 
Afin d'assurer la protection du territoire dans les zones désignées de la région agricole, une commission, la Commission de protection du territoire agricole du Québec, fut instituée. Celle-ci fut immédiatement chargée:

- de décider des demandes d'autorisation qui lui sont soumises en vertu de la loi relativement à l'utilisation, au lotissement ou à l'aliénation d'un lot, de même que des demandes visant à l'inclusion d'un lot dans une zone agricole ou à l'exclusion d'un lot d'une zone agricole;

- de délivrer les permis d'exploitation requis pour l'enlèvement du sol arable conformément à la section $\mathrm{V}$ [de la loi];

- de délimiter, en collaboration avec la municipalité locale, la zone agricole du territoire de celle-ci;

- d'émettre un avis sur toute autre affaire qui doit lui être référée en vertu de la loi.

- de surveiller l'application de la [loi] (Assemblée nationale du Québec 1978j).

La CPTAQ veilla dès lors à ce que l'interdiction de morceler les terres, initialement justifiée par les liens généralement reconnus entre la superficie et l'usage projeté (Union des producteurs agricoles 2018), soit respectée. Pour ce faire, elle assuma l'existence d'une correspondance étroite entre la taille des projets agricoles et leur viabilité, limitant insidieusement les possibilités d'installation dans des secteurs ne nécessitant pas de grandes superficies, comme la culture biologique à petite échelle. À partir de 1989, les concepts de «rentabilité » et de "viabilité » intervinrent plus formellement dans l'interprétation de la notion de «superficie suffisante», nouvellement introduite et fixée à 100 hectares.

L'ensemble des entraves apportées à l'accession à la propriété agricole favorisèrent de ce fait une relève agricole établie sur des exploitations consolidées et lourdement capitalisées. Ne correspondant pas au modèle souhaité d'agriculture "à temps plein » permettant de vivre exclusivement de l'agriculture (Mundler et Ouellet 2017), l'agriculture dite «à temps partiel», pourtant retenue et encouragée par l'OCDE dans les années 1970 (Organisation de coopération et de développement économiques 1978), fut écartée en tant que possibilité.

«Les jeunes, qui bien souvent commencent par pratiquer l'agriculture à temps partiel ne s[eront] ni autorisés à acheter des terres supplémentaires ni à construire une maison sur leur terrain » (Organisation de coopération et de développement économiques 2010, 240).

En arbitrant les demandes d'autorisation qui lui sont soumises (Mundler et Ouellet 2017), la CPTAQ contribua à limiter les possibilités d'installation en agriculture, l'accès à la profession, en plus de définir cette dernière plus précisément que l'avait fait la LPA en recensant l'ensemble des agriculteurs réalisant minimalement $3000 \$$ de recettes brutes avec la vente de leurs produits agricoles, et donc plusieurs exploitations qui avaient une activité non agricole pour activité principale. Maintenant, seuls les producteurs engagés dans un certain volume de production et exerçant cette activité «à temps plein » allaient pouvoir bénéficier des avantages associés à la reconnaissance du statut de producteur (Mundler et Ouellet 2017).

\section{MÉTHODOLOGIE: UNE ANALYSE DE CONTENU}

Notre démarche méthodologique repose sur l'hypothèse selon laquelle les discours et commentaires de la LPTA et de la LPTAA, lors de leur adoption à l'Assemblée nationale du Québec en 1978 et en
1996, sont fondamentaux pour expliquer la genèse d'une nouvelle définition de la profession d'agriculteur au Québec et la façon dont celle-ci s'est construite à travers ces débats. Ils donnent accès à l'ensemble du travail symbolique et social de frontières inscrit au coeur du processus de professionnalisation de l'agriculture québécoise, tel qu'il a donné lieu à l'avènement et la fermeture de la profession au sein du système de la division sociale du travail dans les années 1970. Un processus qui ajouta à l'opposition traditionnelle entre le «bon » et le «mauvais» producteur (Commission Héon 1955), une opposition entre «professionnel » et «non professionnel » (Grignon 1982).

La recherche que nous présentons dans cet article est issue d'une recherche doctorale plus vaste, soutenue par le Conseil de recherches en sciences humaines du Canada $(\mathrm{CRSH})^{3}$, visant à élucider les processus sociaux et spatiaux de la «dépaysanisation » de l'agriculture et de l'espace rural au Québec dans les suites de la Seconde Guerre mondiale (Francoeur 2018). Nous présentons ici une étude qualitative que nous avons conduite à partir d'un corpus formé des verbatim des travaux parlementaires autour des projets de loi 90 (Assemblée nationale du Québec 1978a , 1978b, 1978c, 1978d, 1978e, 1978f, 1978g, 1978h, 1978i, 1978j) et 23 (Assemblée nationale du Québec 1995a, 1995b, 1995c, 1996a, 1996b, 1996c, 1996d), dans laquelle nous mettons en évidence le rôle idéologique de la protection du territoire et des activités agricoles dans la professionnalisation de l'agriculture, de même que dans la limitation de l'accès à la profession. En combinaison avec un examen détaillé des articles pertinents parus dans la presse professionnelle et syndicale (La Terre de chez nous) entre le dépôt du projet de loi 90 le 9 novembre 1978 et le dernier décret intervenu le 7 novembre 1984, cette démarche nous a permis de rendre compte des principaux arguments et des intentions ayant présidé à la mise en place du régime de protection du territoire agricole institué dans les années 1970 au Québec.

Sur le plan technique, nous nous sommes inspirées de la méthode Morin-Chartier (Leray 2008), une méthode d'analyse de contenu qui permet de circonscrire, d'identifier et d'évaluer les différentes unités de sens (appelées unités d'information) à l'intérieur d'un corpus. Suivant cette méthode et s'accompagnant du logiciel NVivo, nous avons procédé à la lecture approfondie de l'ensemble des documents sélectionnés de manière à en découper le contenu en fonction des unités repérées et répertoriées. L'objectif de ce découpage était d'établir sous quel angle (sujets, dossiers) et avec quelles intentions les différents groupes d'intervenants représentés à l'Assemblée nationale - ici, le gouvernement et l'opposition - ont débattu de la protection du territoire (et des activités) agricole(s) à l'occasion des débats entourant l'adoption des projets de loi 90 et 23. Les catégories de la grille d'analyse ont été constituée de manière inductive et sont présentées en détail dans la section suivante; seules les catégories d'intervenants (gouvernement, opposition) et les périodes (1978, 1995-1996) ont été identifiées en amont de la codification. L'identification de périodes a permis de repérer certains changements de ton, de même que différentes évolutions et constances dans les sujets, dossiers et groupes d'intervenants.

Tout au long de l'analyse, les données collectées ont fait l'objet d'une triangulation et de vérifications croisées avec des données du corpus de la sociologie rurale québécoise, l'observation des faits historiques concernant l'évolution effective des conditions d'exercice de la profession d'agriculteur, et des ressources historiographiques (commissions parlementaires, rapports gouvernementaux, etc.).

Nous exposons ici les principaux résultats de cette analyse, en particulier les grands thèmes et idées que contient le corpus, et en dégageons de certaines conclusions. 
ment mises à mal par la saturation de l'espace agraire (Bouchard 1996), et commander de nouvelles stratégies de reproduction fami-

\section{La professionnalisation comme outil de protection du territoire agricole québécois}

À l'occasion de la première lecture du projet de loi 90, Loi sur la protection du territoire agricole, le 9 novembre 1978 à l'Assemblée nationale du Québec, le ministre de l'agriculture de l'époque, Jean Garon, présentait les lignes directrices du projet ministériel:

« M. le Président, le projet de loi sur la protection du territoire agricole a principalement pour objet d'assurer la protection du territoire agricole québécois. À cette fin, il prévoit la mise sur pied d'une commission chargée de surveiller l'application de la loi. Le projet de loi prévoit l'établissement d'une région agricole désignée à l'intérieur de laquelle nul ne pourra, sans l'autorisation de la Commission de la protection du territoire agricole du Québec, poser à l'égard d'un lot certains actes, tels le lotissement, l'utilisation du lot à des fins autres que l'agriculture et l'enlèvement du sol arable pour fins de vente. Cette région agricole désignée comprendra une partie des basses terres du Saint-Laurent et de l'Outaouais. D'autres régions agricoles désignées pourront être établies par décret du gouvernement» (gouvernement, Assemblée nationale du Québec 1978a).

Reprenant la parole le 16 novembre lors de la deuxième lecture, Jean Garon précisait les intentions du gouvernement en matière de protection du territoire agricole:

« II faut empêcher que se constituent des petites fermes non rentables, des petites fermes d'amateurs, pour que cela soit vraiment des fermes d'agriculteurs. [...] il ne faut pas que les terres soient trop petites. II faut, dans une zone agricole, des objectifs d'utilisation agricole du sol, de consolidation des exploitations et au moins le maintien des exploitations agricoles existantes» (gouvernement, Assemblée nationale du Québec 1978i).

Afin de limiter le nombre de petites fermes, d'assurer des possibilités d'expansion aux fermes existantes et d'assurer une relève agricole sur des terres consolidées, le projet de loi interdisait le morcellement des terres en zone protégée. Ce faisant, le projet limitait l'accès à la profession de producteur agricole en ciblant un nouvel élément de professionnalisation, ignoré par la LPA quelques années plus tôt: l'existence d'une superficie minimale en-deçà de laquelle il n'était plus possible de pratiquer l'agriculture de façon reconnue. Le contrôle du morcellement des terres impliquait en outre une vision de l'agriculture qui entrait en conflit avec les pratiques associées à la paysannerie traditionnelle, tel que le soulignait l'opposition à l'Assemblée nationale à l'occasion de la deuxième lecture:

« [E] lle [la loi] vient imposer des restrictions discriminatoires à l'endroit de ceux qui possèdent le sol par rapport aux traditions qu'on a toujours connues chez les propriétaires du sol au Québec. On ignore totalement la personne. Non seulement on l'ignore, mais on la brime dans des droits que je qualifie de fondamentaux, des droits de propriété qui ont toujours été la marque dominante de la possession de la terre, de la possession du bien agricole » (opposition, Assemblée nationale du Québec 1978i).

Les nouvelles modalités concernant l'indivisibilité du foncier agricole menaçaient certaines pratiques paysannes traditionnellement associées au processus de la reproduction familiale. Le projet de loi venait complexifier les modalités du pluri-établissement, déjà forte- liale davantage en lien avec la notion de patrimoine et de consolidation.

«Le vrai cultivateur n'a même plus le loisir ou la liberté de donner une petite parcelle, un lot sur sa ferme à l'un ou l'autre de ses enfants» (opposition, Assemblée nationale du Québec 1978i).

«On lui enlève le droit de partager librement, par testament, même pour les fins d'agriculture, son bien immobilier, sa terre et ses bâtiments, à parts égales entre ses enfants, ce projet de loi rendant une propriété agricole non morcelable, sauf pour agrandir les propriétés agricoles des voisins » (opposition, Assemblée nationale du Québec 1978d).

Le processus marqua une étape déterminante dans la professionnalisation du métier, du moins dans sa dimension territoriale, tout en poursuivant et intensifiant les débats autour de la définition de ce qu'est un producteur agricole. À ce titre, le ministre de l'agriculture assurait que les «vrais agriculteurs », ceux qui faisaient «de la vraie culture», ne cherchaient pas à morceler les terres agricoles (gouvernement, Assemblée nationale du Québec 1978g). Ils cherchaient au contraire à les agrandir, de manière à en dégager un revenu suffisant:

«[L]es gens ne veulent pas subdiviser ou rapetisser leur morceau de terre. Au contraire, ils tendent à les agrandir parce que les tracteurs sont de plus en plus gros et qu'on cultive des espaces toujours de plus en plus grands. Donc, je ne pense pas que ce projet de loi brime les cultivateurs qui voudraient léguer leur terre à leurs fils. S'ils veulent léguer leur terre à leurs fils, ils vont laisser la terre au complet à un ou deux de leurs fils qui se formeront en compagnie et ces fils-là pourront peut-être même acheter la terre du voisin pour pouvoir cultiver un plus grande superficie» (gouvernement, Assemblée nationale du Québec 1978h).

«[C]e n'est pas souhaitable que la terre soit divisée, soit en petits lopins et en petits morceaux de cinq ou dix acres parce qu'aucun des enfants ne va pouvoir gagner sa vie sur la terre» (gouvernement, Assemblée nationale du Québec 1978d).

Alors que le ton montait à l'Assemblée nationale, les catégories de «vrais » et de «véritables » agriculteurs étaient indistinctement mobilisées par le gouvernement et l'opposition et ce, de manière parfois explicite, parfois implicite. Elles figuraient au coeur du débat.

«Ce projet de loi [...] ne donne aucune garantie que le sol agricole demeurera la propriété des vrais exploitants, c'està-dire les agriculteurs. En effet, toute personne désireuse d'acquérir un sol arable peut le faire en tout temps, même si elle est non-résidente. Quant aux citadins, un citadin pourra devenir propriétaire d'un sol arable à condition qu'il achète au complet une terre » (opposition, Assemblée nationale du Québec 1978e).

"Celui qui a de l'argent peut s'acheter une ferme au complet, il peut la garder en friche, il n'y a pas de problème. Notre loi lui donne ces pouvoirs et reconnaît cela» (opposition, Assemblée nationale du Québec 1978i). 
L'opposition exigeait davantage de mesures contre ceux qu'on appelait les «gentlemen farmers ${ }^{4}$ », tout en dénonçant que l'on chasse du territoire rural «des personnes qui autrement auraient été intéressées à y demeurer encore longtemps» (opposition, Assemblée nationale du Québec 1978i).

«[I] faudra que vous voyiez aux "gentlemen farmers» aussi, que vous les arrêtiez de spéculer! Des gars qui ont trois boeufs et deux cochons et qui sont professeurs d'université. Une minute, là! Mon cher monsieur, il va falloir que vous y voyiez! » (opposition, Assemblée nationale du Québec 1978e)

\section{La concentration et la protection de l'accès à l'aide de l'État sur les terres « protégées »}

Le 21 novembre 1978, à l'occasion de la deuxième lecture du projet de loi 90 à l'Assemblée nationale, l'adjoint parlementaire au ministre de l'agriculture - Réal Rancourt - soulignait l'importance de la protection du territoire agricole dans la stabilité de l'agriculture, régulant la valeur des fermes et stabilisant les prix:

«Le terme «stabilité » devient d'ailleurs, à mon avis, un mot de passe en regard de ce projet de loi : stabilité pour la relève agricole, pour ces jeunes filles et garçons motivés et instruits dans cette technique qu'est l'agriculture; stabilité des revenus; stabilité de l'écoulement des productions qui pourront être planifiées dans le temps et permettront de négocier un coût de production réaliste dans l'approvisionnement des Québécois citadins qui seront les grands gagnants » (gouvernement, Assemblée nationale du Québec 1978h).

L'une des principales critiques adressées au projet de loi 90 concernait pourtant l'absence de politique agricole. En dehors d'une augmentation du remboursement des taxes foncières des producteurs reconnus et situés dans la zone agricole, le projet de loi ne prévoyait aucune mesure pour rentabiliser l'agriculture et «consolider la structure de ferme idéale» (opposition, Assemblée nationale du Québec 1978d) envisagée par le gouvernement. Rien dans le contenu du projet de loi, n'indiquait que «le territoire agricole sera[it] mieux utilisé » (opposition, Assemblée nationale du Québec 1978i) suivant l'adoption de la loi.

L'opposition réclamait que la loi soit assortie d'aides à l'agriculture, et que celles-ci soient concentrées sur les terres «protégées ». L'argent devait aller aux «véritables » agriculteurs, et la relève devait être en mesure de s'installer en agriculture (opposition, Assemblée nationale du Québec 1978d). Pour ce faire, il fallait réviser le seuil d'admissibilité au statut de producteur agricole, tel que fixé dans la LPA.

«[I] faut cesser de subventionner de faux agriculteurs ou des agriculteurs de fin de semaine. II y en a 9000 ou 10 000 au Québec à l'heure actuelle qui monopolisent les subventions ou l'aide gouvernementale qui devrait aller aux véritables agriculteurs québécois » (opposition, Assemblée nationale du Québec 1978g).

«[C]e rabais [le remboursement des taxes foncières] s'adresse indistinctement aux vrais agriculteurs comme aux agriculteurs de fin de semaine qui ont le statut de producteurs agricoles pour autant qu'ils ont $1000 \$$ de revenus par année ou plus. Ces derniers dont on peut évaluer le nombre entre 9000 et 10000 au Québec privent nos producteurs agricoles de sommes d'argent qui devraient être consacrées aux professionnels de l'agriculture et non aux professionnels urbains en vacances à la campagne, ce qu'on appelle les «gentlemen farmers » » (opposition, Assemblée nationale du Québec 1978i).

L'extrait suivant, tiré de l'édition du 5 octobre 1978 de l'hebdomadaire agricole La Terre de chez nous, illustre bien cette stratégie qui visait à limiter «le nombre d'appelés au partage du gâteau » et à augmenter «la part dévolue à chacun », de manière à favoriser la parité professionnelle revendiquée:

« [L]e zonage, comme le contingentement, limiterait l'accès à la profession, créerait une rareté relative de l'outil qu'est la terre et du coup le valoriserait. Les sols, comme les quotas, deviendraient des biens rares et donc précieux [...]. La concentration sur les seules terres protégées de l'aide de l'État, en réduisant le nombre d'appelés au partage du gâteau, augmenterait la part dévolue à chacun» (Charbonneau 1978).

Avec l'absence d'aide de l'État sur les terres «protégées », l'autorisation de l'utilisation des friches agricoles en territoire zoné - laquelle participera à une problématique autour des terres abandonnées - contribua à entourer d'incertitude le véritable objectif visé par le projet de loi. L'existence d'un surplus de terres agricoles fut ainsi soulevée par l'opposition, ainsi que l'avait fait la Commission de planification de l'agriculture canadienne (1969) quelques années plus tôt5.

"It is suggested by this government that there is a great crisis in arable land in the province of Quebec. And so, we must impose the great act of saving this arable land, by imposing agriculturals only. Madam President, first of all, let us examine: Is there a crisis in arable land in the province of Quebec?" (opposition, Assemblée nationale du Québec 1978h)

«Ce n'est pas le manque de terres, c'est le manque de politiques de développement de l'agriculture qui déçoit les agriculteurs. La preuve en est que des centaines de milliers d'acres de bonnes terres sont en friche au Québec» (opposition, Assemblée nationale du Québec 1978h).

En particulier, Fabien Roy, député du Parti national populaire et membre de l'opposition, souleva la contradiction entre la politique de zonage qui incluait d'importantes superficies non cultivées (car laissées en friche), d'une part, et le contingentement de la production, d'autre part:

«[L]'agriculture au Québec fonctionne de plus en plus à partir de quotas, s'il est impossible d'obtenir un quota de production, qu'est-ce qu'on va faire des territoires agricoles protégés?» (opposition, Assemblée nationale du Québec 1978e)

La protection du droit de produire et la création d'une immunité à l'égard des poursuites

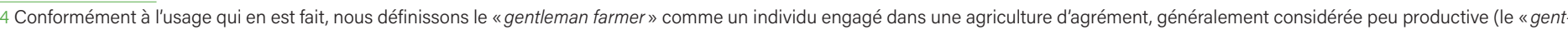
leman farmer » dispose de revenus indépendamment de sa pratique agricole); ici, la citation réfère aux professeurs universitaires, qui ne dépendent pas de l'agriculture pour vivre.

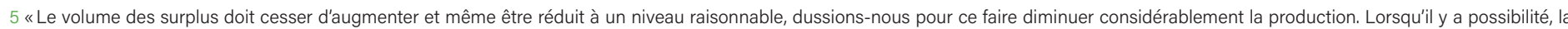

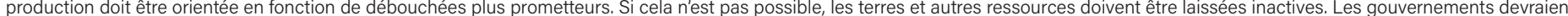

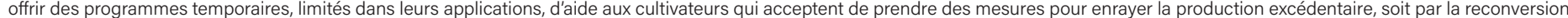
de leurs exploitations, soit par l'abandon de terres agricoles» (Commission de planification de l'agriculture canadienne 1969).
} 
Le 16 novembre 1978, lors de la deuxième lecture du projet de loi 90 à l'Assemblée nationale, Jean Garon décrivait la méthodologie de protection du territoire agricole retenue par le gouvernement:

«La technique de protection prévue par le projet de loi permet d'interdire toute utilisation du sol inconciliable avec l'agriculture à l'intérieur de ce qui paraît être un territoire viable pour l'agriculture» (gouvernement, Assemblée nationale du Québec 1978i).

En particulier, le gouvernement visait l'implantation de résidences à proximité des exploitations agricoles, «le long des routes ou des rangs » (gouvernement, Assemblée nationale du Québec 1978i). Reprenant un argument déjà avancé par le comité d'enquête pour la protection des agriculteurs et des consommateurs en 1955, il fondait son intervention sur la séparation des fonctions agricoles et non agricoles, « en portant tout particulièrement la charge contre l'implantation résidentielle» (Côté, Mercier, and Roy 2014, 400). II était temps, disait-on, «que les citadins cessent d'importuner indûment les agriculteurs » (opposition, Assemblée nationale du Québec 1978i).

Alors que différents jugements avaient menacé la primauté accordée à l'agriculture en zone agricole (Côté, Mercier, and Roy 2014) ${ }^{6}$, un avant-projet de loi - l'avant-projet de loi modifiant la LPTA et d'autres dispositions législatives afin de favoriser la protection et le développement durable des activités agricoles - chercha bientôt à renforcer la préséance de l'agriculture vis-à-vis de toute activité inconciliable ou incompatible. Déposé à l'Assemblée nationale le 22 juin 1995 (Assemblée nationale du Québec 1978a), l'avant-projet 23 visait à reconnaitre un «droit de produire » aux producteurs agricoles, un droit qui, une fois reconnu, mettrait ceux-ci à l'abri de toute poursuite relative à leur pratique professionnelle.

Rejoignant une préoccupation des industriels de l'élevage (porc, volaille), l'avant-projet visait, par le «développement durable», la cohabitation harmonieuse entre les activités agricoles et les autres fonctions du territoire rural, dans un contexte où la tendance était à la diversification des activités et des usages (agriculture, agroforesterie, agrotourisme, etc.). Cette coexistence devait premièrement reposer sur la reconnaissance d'une différence entre la pollution d'origine agricole relevant du règlement sur l'eau et le sol, et les nuisances et inconvénients de voisinage résultant d'activités agricoles normales (air). Dans l'avant-projet de loi, la question de ce qu'est une pratique agricole «normale» devait être résolue par la création d'un guide des pratiques agricoles. Ce guide devait avoir pour objectifs la reconnaissance des pratiques professionnelles des producteurs agricoles afin que leurs partenaires et voisins «comprennent» que certains inconvénients sont inhérents à une pratique agricole «normale » (1), de même que le développement de mesures d'atténuation des effets sur l'environnement et le voisinage de pratiques agricoles qui dépassent le cadre des pratiques normales (2). La préparation de ce guide devait initialement impliquer le monde agricole, de même que le monde municipal, le secteur de l'environnement et celui de la santé publique. II devait être périodiquement révisé afin de refléter le caractère évolutif des pratiques agricoles professionnelles.

Dans la version subséquente du projet de loi, présentée à l'Assemblée nationale le 15 mai 1996, le guide devait cependant perdre sa fonction centrale dans l'application de la loi et ne devenir qu'un outil secondaire à l'usage du médiateur?. La création du guide devait finalement laisser place à la formulation d'orientations gouvernementales à partir desquelles les municipalités régionales de comté (MRC) allaient devoir réviser leur schéma d'aménagement, outil introduit par la Loi sur l'aménagement et l'urbanisme en 1979. Ces orientations devaient porter sur différentes normes en matière d'odeurs (au moyen de distances séparatrices), de bruits et de poussières. Le faible degré de sévérité des normes en matière de développement durable devait cependant susciter différents questionnements quant au développement véritablement durable des activités agricoles que l'on souhaitait favoriser par le biais dudit projet de loi. Au nom de l'acception du développement durable contenue dans le projet de loi 23, celui-ci devait conférer une immunité aux producteurs agricoles en matière de nuisances et inconvénients de voisinage résultant d'activités agricoles «normales» (bruits, poussières, odeurs). Cette immunité ne devait être subordonnée qu'au respect des normes règlementaires provinciales en vertu de la Loi sur la qualité de l'environnement (bruits et poussières), ainsi qu'au respect des règlements municipaux en matière d'odeurs. En commission parlementaire, cette exonération devait être vertement critiquée par différents groupes pour créer un précédent abusif et dangereux, ainsi qu'une entorse au principe d'égalité des citoyens devant la loi. Il devait en être de même pour le statut privilégié de l'UPA dans les débats.

La primauté accordée à l'agriculture sera finalement inscrite au coeur de la LPTAA. En particulier, l'article 62 exposera l'ensemble des critères sur la base desquelles la CPTAQ, chargée de l'application de la loi, devra faire reposer ses décisions. «De ces dix critères, les huit premiers considère[ront] que les activités non agricoles n'ont de légitimité en zone agricole que si elles ne nuisent pas à une activité agricole actuelle ou potentielle» (Côté, Mercier, and Roy 2014, 398).

\section{Le rôle d'arbitrage de la CPTAQ}

Comme la RMAAQ à laquelle le ministre Toupin avait confié la fonction d'assurer la protection du statut de producteur agricole en 1972, la CPTAQ se vit chargée de l'application de la loi sur la protection du territoire agricole en 1978. En commission parlementaire, on accusa donc le gouvernement de récupérer des pouvoirs de protection du territoire agricole appartenant aux municipalités et d'ainsi porter atteinte à l'autonomie municipale et, par le fait même, à la liberté de choix.

«C'est un projet de loi qui est un accroc à la liberté de choix du milieu de vie des Québécois. C'est un projet de loi qui se substitue d'une manière absolue et radicale aux 614 municipalités du Québec affectées en ce qui concerne leur planification et le développement de leur territoire» (opposition, Assemblée nationale du Québec 1978d).

Les pouvoirs discrétionnaires et arbitraires de la CPTAQ furent par ailleurs au centre de la critique. En retirant au monde municipal la gestion d'une partie de son territoire, la Commission s'arrogeait des pouvoirs décisionnels exclusifs en matière de zonage agricole et donc, de pratique agricole. De plus, les critères qui, selon le texte du projet de loi, devaient présider à ses décisions dans l'exercice de ses fonctions furent soumis au doute: allait-on obliger la future commission à prendre en considération l'avis des municipalités dans l'exercice de ses fonctions? Allait-on moduler ou uniformiser l'application de la loi dans l'ensemble des régions?

« [N]ous ne pouvons accepter l'abus de pouvoirs dont cette commission serait investie. Elle seule possède tous les pouvoirs décisionnels et elle seule émet des ordonnances. Cette commission est donc juge et partie et exercera des pouvoirs dictatoriaux» (opposition, Assemblée nationale du Québec 1978i).

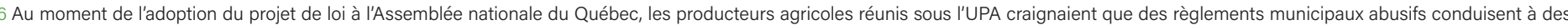

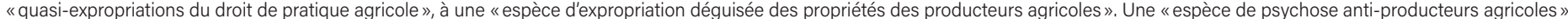
était, disait-on, en train de se bâtir.

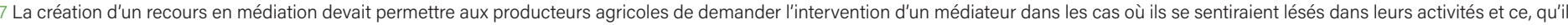
s'agisse ou non de pratiques considérées comme «normales».
} 
La création de la zone agricole allait par ailleurs obéir à des critères critiqués pour leur faible scientificité, peu sensibles à la diversité des situations régionales. À ce titre, l'opposition demanda à ce que la politique de zonage s'inscrive "dans un plan global d'utilisation et d'aménagement du territoire » (opposition, Assemblée nationale du Québec 1978i) et à ce qu'elle s'applique à l'ensemble du territoire québécois, de manière à ne pas créer «deux sortes d'agriculture» dans la province:

«Ce sont toutes des façons, M. le Président, pour faire deux sortes d'agriculture: l'agriculture à l'intérieur et l'agriculture à l'extérieur; les régions considérées prioritairement comme agricoles et les régions périphériques qui sont laissées pour compte» (opposition, Assemblée nationale du Québec 1978d).

Bien que le ministre Garon admît que le syndicalisme agricole souhaitait que les mesures de protection soient étendues à l'ensemble du Québec (gouvernement, Assemblée nationale du Québec 1978i), il suggéra que l'application de la loi devait toutefois faire l'objet de mesures de modulation régionale, qui viendraient en temps et lieux:

«Des gens qui m'ont dit à peu près la même chose: de protéger les terres. Sauf dans le Bas-Saint-Laurent et la Gaspésie où ils m'ont dit que leur problème était principalement l'abandon des terres, parce que les gens gagnaient difficilement leur vie, qu'ils avaient des difficultés et qu'il y avait le problème de l'abandon des terres. La protection des terres dans leur région devait donc se faire de façon différente» (gouvernement, Assemblée nationale du Québec 1978d).

Aujourd'hui encore, quarante ans après l'adoption de la LPTA en 1978, et malgré ses nombreuses révisions, l'application de la loi ne prévoit aucune mesure de modulation régionale et s'applique uniformément à l'ensemble des régions.

\section{UNE PROFESSION OBJET DE CONFLITS}

Notre analyse s'intéresse à la place du zonage agricole dans la professionnalisation de l'agriculture québécoise, de même que dans la limitation de l'accès à la profession (1), ainsi qu'à ses conséquences pour le développement et le renouvellement de l'agriculture aujourd'hui (2). Elle interroge le caractère politique et idéologique de la protection du territoire et des activités agricoles en révélant l'importance de l'espace et des modalités de son occupation dans les processus de la professionnalisation de l'agriculture au Québec.

Au terme de cette analyse, nous pensons pouvoir dire avec suffisamment de certitude qu'à la fin des années 1970, la loi sur la protection du territoire agricole est venue s'inscrire dans la continuité de l'objectif global de la profession et du gouvernement de l'époque, qui est de professionnaliser l'agriculture. En conférant à l'espace une fonction exclusivement agricole, la loi a institutionnalisé sur le plan spatial l'intégration de l'agriculture au système de la division sociale du travail, tout en permettant à un nombre tendanciellement restreint de producteurs d'obtenir (du moins virtuellement) la maitrise de l'espace rural. Le géographe Henri Lefebvre (1974) a déjà montré que plus un espace est fonctionnalisé, moins il se prête à l'appropriation.

Les promoteurs du modèle espéraient que cet exercice de zonage retire de la production agricole des pans suffisants du territoire pour permettre aux producteurs restants d'accéder à la parité professionnelle (La Terre de chez nous 1967), qui était au cœur des préoccupations de la profession et du gouvernement. La désignation d'une superficie minimale à la pratique de l'agriculture (et ce, que les superficies soient cultivées ou non) devait organiser les conditions d'une mise en concurrence pour la terre. Les concepts de « rentabilité » et de "viabilité » intervenant dans l'interprétation de la notion de «superficie suffisante» devaient en outre laisser peu de place à une diversité de modes d'entreprise agricole, dont les productions ne nécessitant pas de grandes superficies. Lors des débats précédant l'adoption de la loi, on souligna les répercussions de ces restrictions pour les pratiques associées à la paysannerie traditionnelle, de même que pour les propriétaires de type "gentlemen farmer», contre lesquels on exigeait davantage de mesures.

À la façon des quotas (qui constituent également un enjeu d'accès à la profession pour la relève agricole), la concentration des aides à l'intérieur de la zone agricole désignée devait « réduire le nombre d'appelés au partage du gâteau » et «augmenter la part dévolue à chacun » (Charbonneau 1978), favorisant la parité professionnelle revendiquée. Pour avoir désormais accès aux aides de l'État en tant que producteur agricole, il fallait être situé en territoire agricole et répondre aux exigences de la LPA adoptée en 1972: vendre un montant minimum de 3000 dollars annuellement.

Il serait impossible de conclure sans souligner le conflit agriculture et environnement, tel qu'il prit forme avec la mise en modèle professionnelle de l'agriculture québécoise dans les années soixante-dix et tel qu'il entend aujourd'hui être résolu par de nouvelles agricultures. En effet, l'analyse thématique des verbatim des travaux parlementaires autour du projet de loi 23 (et de l'avant-projet) nous a permis de rendre compte de l'offensive menée par le secteur et le syndicalisme agricoles face à la dégradation du lien contractuel entre l'agriculture et le reste de la société québécoise dans les années quatrevingt-dix, alors que se structuraient et se multipliaient les initiatives citoyennes dans les régions rurales où l'industrie porcine se concentrait (à commencer par le Bas-Saint-Laurent). Très controversée, l'industrie était alors dénoncée pour ses «ravages » sur l'environnement. En réponse aux préoccupations que les initiatives citoyennes (et que les premiers jugements) faisaient naitre chez les producteurs agricoles concernés (en particulier, dans le porc, mais aussi dans la volaille), le projet de loi 23 permit aux producteurs agricoles d'obtenir la reconnaissance officielle de leur pratique professionnelle. Face à la menace réelle ou fictive d'expropriations du droit de pratique et des terres agricoles, ce projet de loi agit sinon en revoyant une pratique professionnelle controversée, du moins en encadrant les pouvoirs réglementaires des municipalités. L'immunité professionnelle conférée aux producteurs agricoles et les dispositions visant la défense de leurs intérêts introduites par le projet de loi 23 (médiation, etc.) défièrent l'idée même d'association professionnelle, dont le mandat aurait été de surveiller les activités professionnelles de ses membres susceptibles de comporter des risques pour le public.

Si l'acception retenue du développement durable devait chercher à entretenir des relations plus harmonieuses entre les différentes fonctions du territoire, le concept de droit de produire entériné par la révision de 1996 élimina autrement toute forme de concurrence en termes d'usage du territoire agricole, par un procédé de «mise à distance» (Côté, Mercier, and Roy 2014). À ce titre, le renforcement du concept d'homogénéité du territoire agricole, introduit par la loi de 1978 sous la forme d'un critère décisionnel, permit la constitution de domaines agricoles suffisamment grands pour éviter toute cohabitation de fonctions jugées incompatibles avec la pratique des activités agricoles, à commencer par la fonction résidentielle. De ce fait, l'espace de cohabitation initialement visé par le projet de loi laissa place à un espace «homogène », qui allait dissimuler, sous son apparente homogénéité, des rapports de domination entre ceux qui maitrisent l'espace et les autres (Lefebvre 1974). À l'échelle nationale, la possibilité d'une modulation régionale dans l'application de la loi fut réfutée au nom de ce même critère d'homogénéité, et la protection du territoire et des activités agricoles s'appliqua uniformément à l'ensemble 
du territoire désigné, indépendamment de la diversité des situations locales et des nombreuses recommandations contraires formulées par les différentes autorités nationale (Commission de l'Agriculture, des Pêcheries, de l'Énergie et des Ressources Naturelles 2015) et internationale (Organisation de coopération et de développement économiques 2010), qui proposent un renforcement dans la plaine de Montréal et la vallée du Saint-Laurent, ainsi qu'un assouplissement ailleurs.

À la fin des années 1990, alors qu'était discuté le projet de loi 23 à l'Assemblée nationale, la participation du secteur environnemental dans les débats politiques marquait une étape symbolique de la mise en œuvre du développement durable au Québec. Contrairement à l'étude du projet de loi 90, l'étude du projet de loi 23 incluait trois organisations du secteur de la protection de l'environnement, dont deux situées dans la région administrative du Bas-Saint-Laurent et une d'envergure nationale. La place de l'environnement dans les débats fut cependant limitée face à l'importance que l'UPA y prit.

Sur la question des implications de ce cadre législatif pour le renouvellement de l'agriculture québécoise aujourd'hui, notons que la périurbanisation touche des territoires toujours plus éloignés des centres urbains, et que les demandes de diversification des activités à la ferme et de pluriactivité se multiplient, notamment dans les régions qui ne sont pas fonctionnellement liées à des centres urbains (Organisation de coopération et de développement économiques 2010). La diversification de l'espace rural semble vouloir favoriser les conditions propres à une agriculture dite «à temps partiel», de sorte que la pluriactivité, par-delà ses figures classiques (la sylviculture), revêt des formes restées jusqu'à maintenant inédites. En particulier, et bien que la présence émergente de nouveaux acteurs ait été récemment confirmée par Pronovost (2016) dans son rapport sur le vécu et les attentes de la relève agricole et des jeunes agriculteurs québécois, les récentes flambées des prix des terres agricoles laissent actuellement peu de possibilités à l'expression de nouvelles formes d'agriculture à petite échelle en dehors de quelques régions où le cout des terres n'excède toujours pas les profits à en escompter (Mauricie, Bas-Saint-Laurent, Lanaudière, SaguenayLac-Saint-Jean, notamment). Même dans ces régions, cependant, l'allocation des places continue de limiter fortement les emplacements que ces nouveaux acteurs sont susceptibles d'occuper. En dépit des révisions successives de la LPTA et de premières prises en compte des particularités régionales dans l'application de la loi (Sanscartier 2011), celle-ci demeure rarement favorable à leur projet. Si quelques jeunes maraichers sur petite surface ont récemment réussi à convaincre la $\mathrm{CPTAQ}^{8}$, celle-ci continue généralement de manquer d'attention quant au potentiel des exploitations de petite taille, indépendamment du profil des requérants et des projets (Pronovost 2016). Les concepts de rentabilité et de viabilité intervenant dans l'interprétation de la notion de «superficie suffisante» continuent d'avantager les «grandes» entreprises, tout en laissant peu de place aux productions nécessitant de petites surfaces. Même s'il est de plus en plus admis que la surface suffisante à la pratique de l'agriculture peut varier, la profession se dit ouverte à une diminution de la superficie suffisante dans l'unique mesure où cette diminution s'accompagne d'un renforcement du droit de produire (Union des producteurs agricoles 2015).

Différentes initiatives peuvent désormais améliorer la possibilité de s'établir dans des productions non contingentées. C'est le cas de la banque de terres du MAPAQ et de la Fédération de la relève agricole du Québec, du Fonds d'investissement pour la relève agricole (FIRA), des fiducies foncières agricoles, des ententes de location, du projet de la Ferme 59 de la MRC de l'Érable, nommé ainsi en réfé- rence à l'article de la LPTAA en vertu duquel une MRC ou une communauté peut présenter une demande à portée collective portant sur la gestion de la fonction résidentielle dans des ilots déstructurés de la zone agricole. Des jeunes cherchent aussi à se réapproprier et à remettre en culture des terres abandonnées dans la période suivant l'adoption de la loi, et certains modèles d'incubateurs d'entreprises agricoles permettent depuis peu le démarrage d'entreprises innovantes sur petite surface (par exemple, la Ferme Bord-du-lac).

Or, le régime de protection du territoire agricole ne tient toujours pas compte des tendances actuelles dans les secteurs agricole, agroalimentaire et agrotouristique, pas plus qu'il ne rend compte de la complexité du territoire qu'il entend protéger (Organisation de coopération et de développement économiques 2010): à défaut d'obtenir l'approbation de la Commission, il demeure interdit d'y établir un atelier de transformation. Le traitement des demandes au cas par cas préconisé se veut par ailleurs rarement favorables aux projets de ce type, en plus de mener à des incohérences dans la gestion du territoire agricole (Ouimet 2015; Lebeau 2015).

De consultation publique en consultation publique (Commission sur l'avenir de l'agriculture et de l'agroalimentaire québécois 2008; Ouimet 2009; Organisation de coopération et de développement économiques 2010; Commission de l'Agriculture, des Pêcheries, de l'Énergie et des Ressources Naturelles 2015), le modèle québécois en matière de protection des terres agricoles est donc critiqué pour son manque de souplesse (absence de modulation régionale), de même que pour son inactualité face aux nouvelles tendances dans le secteur (pluriactivité, durabilité des espaces ruraux, agriculture dite «à temps partiel»). Loin d'être assuré, le développement de nouvelles formes d'agriculture à petite échelle renvoie donc encore aujourd'hui à une occupation dynamique, mais irrégulière, du territoire et à un exercice tout aussi irrégulier, parfois même hors-la-loi, du métier. Tout en entendant résoudre le conflit agriculture et environnement, ces nouvelles agricultures prennent la forme de luttes dans lesquelles la pluriactivité continue de permettre à un nombre croissant d'individus de contourner le modèle de base du professionnalisme agricole et de revendiquer des pratiques alternatives de production, de financement et de commercialisation, davantage en accord avec certaines pratiques associées à la paysannerie traditionnelle. Ce faisant, elles mettent de l'avant des pratiques qui vont à l'encontre des priorités défendues par les lois 90 et 23 dans le contexte de leur adoption à l'Assemblée nationale. Porteuses d'une compréhension renouvelée de ce que devrait être la protection du territoire et des activités agricoles, ces agricultures évoluent sous le signe de la durabilité écologique, de l'occupation dynamique du territoire, du tournant vers la qualité, de la valeur ajoutée, de la diversification et de la relocalisation.

Le processus de professionnalisation a paradoxalement légèrement accru le nombre des propriétaires d'exploitations agricoles de petite taille exerçant l'agriculture «à temps partiel», combinant l'exercice de l'agriculture à celui d'une profession libérale (Pronovost 2016), ou faisant de l'agriculture l'objectif d'un changement de carrière. Révélatrices des processus en cours, ces initiatives font écho aux métamorphoses, dans l'agriculture comme dans les autres secteurs économiques, des modes d'exercice de l'activité professionnelle (Blanchemanche et al. 2000), qui ne correspondent plus nécessairement à l'emploi monoactif «à temps plein». Si toutes ne portent pas les mêmes ambitions de développement et de professionnalisation, il devient toutefois difficile de les ignorer. Dans un contexte où la disparité des revenus agricoles se maintient et que les écarts se creusent au sein de la profession organisée, la situation de la plupart des agriculteurs professionnels demeure «peu enviable» (Nan-

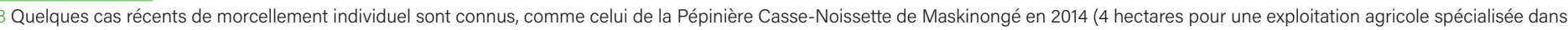

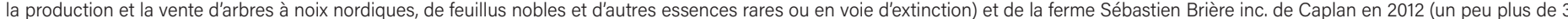

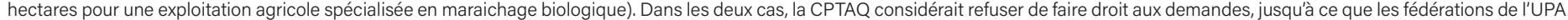
de la Mauricie et de la Gaspésie-Les-Îles les appuient respectivement (SOQUIJ, 2016).
} 
tel 2015) et invite à examiner la possibilité de nouvelles formes de professionnalité agricole, voire de mesures de modulation régionale. Une géographie prospective permettrait certainement d'imaginer différentes possibilités d'évolution pour le territoire agricole, suivant ses transformations historiques, contemporaines et à venir, de même que celles du rapport au travail et à l'environnement.

\section{BIBLIOGRAPHIE}

Assemblée nationale du Québec. 1978a. «Journal Des Débats de L'Assemblée Nationale,» http://www.assnat.qc.ca/fr/travaux-parlementaires/assemblee-nationale/31-3/journal-debats/19781116/121623.html.

—. 1978b. «Journal Des Débats de L'Assemblée Nationale.» http:// www.assnat.qc.ca/fr/travaux-parlementaires/assemblee-nationale/31-3/journal-debats/19781221/121663.html.

—. 1978c. «Journal Des Débats de L'Assemblée Nationale.» http:// www.assnat.qc.ca/fr/travaux-parlementaires/assemblee-nationale/31-3/journal-debats/19781220/121661.html.

—. 1978d. «Journal Des Débats de L'Assemblée Nationale.» http:// www.assnat.qc.ca/fr/travaux-parlementaires/assemblee-nationale/31-3/journal-debats/19781204/121639.html.

—. 1978e. «Journal Des Débats de L'Assemblée Nationale.» http:// www.assnat.qc.ca/fr/travaux-parlementaires/assemblee-nationale/31-3/journal-debats/19781201/121637.html.

—. 1978f. «Journal Des Débats de L'Assemblée Nationale.» http:// www.assnat.qc.ca/fr/travaux-parlementaires/assemblee-nationale/31-3/journal-debats/19781128/121631.html.

—. 1978g. «Journal Des Débats de L'Assemblée Nationale.» http:// www.assnat.qc.ca/fr/travaux-parlementaires/assemblee-nationale/31-3/journal-debats/19781122/121627.html.

—. 1978h. «Journal Des Débats de L'Assemblée Nationale.» http:// www.assnat.qc.ca/fr/travaux-parlementaires/assemblee-nationale/31-3/journal-debats/19781121/121625.html.

—. 1978i. «Journal Des Débats de L'Assemblée Nationale.» http:// www.assnat.qc.ca/fr/travaux-parlementaires/assemblee-nationale/31-3/journal-debats/19781116/121623.html.

—. 1978j. «Loi Sur La Protection Du Territoire et Des Activités Agricoles, » http://legisquebec.gouv.qc.ca/fr/ShowDoc/cs/P-41.1.

—. 1995a. «Journal Des Débats de L'Assemblée Nationale.» http:// www.assnat.qc.ca/fr/travaux-parlementaires/assemblee-nationale/35-1/journal-debats/19951128/6577.html.

—. 1995b. «Journal Des Débats de L'Assemblée Nationale.» http:// www.assnat.qc.ca/fr/travaux-parlementaires/assemblee-nationale/35-1/journal-debats/19950920/6575.html.

—. 1995c. «Journal Des Débats de L'Assemblée Nationale.» http:// www.assnat.qc.ca/fr/travaux-parlementaires/assemblee-nationale/35-1/journal-debats/19950622/6559.html\#950622003.

—. 1996a. «Journal Des Débats de L'Assemblée Nationale.» http:// www.assnat.qc.ca/fr/travaux-parlementaires/assemblee-nationale/35-2/journal-debats/19960619/6687.html.

—. 1996b. «Journal Des Débats de L'Assemblée Nationale.» http:// www.assnat.qc.ca/fr/travaux-parlementaires/assemblee-nationale/35-2/journal-debats/19960611/6675.html.

-_-. 1996c. «Journal Des Débats de L'Assemblée Nationale.» http://www.assnat.qc.ca/fr/travaux-parlementaires/assemblee-nationale/35-2/journal-debats/19960604/6665.html.
—. 1996d. «Journal Des Débats de L'Assemblée Nationale. » http:// www.assnat.qc.ca/fr/travaux-parlementaires/assemblee-nationale/35-2/journal-debats/19960515/6647.html\#960515049.

Blanchemanche, S., Laurent, C., Mouriaux, M. F., \& Peskine, E. (2000). Multifonctionnalité de l'agriculture et statuts d'activité. Économie rurale, 260(1), 41-51.

Bouchard, Gérard. 1996. Quelques Arpents d'Amérique: Population, économie, Famille Au Saguenay, 1838-1971. Editions du Boreal.

Bouchard, R. (2014). Les champs de bataille: histoire et défis de l'agriculture biologique au Québec. Les Éditions Écosociété.

Charbonneau, André. 1978. «Le Zonage Agricole: Un Défi Plus Politique Qu'agricole. » La Terre de Chez Nous, 3.

Commission de l'Agriculture, des Pêcheries, de l'Énergie et des Ressources Naturelles. 2015. Examen Des Orientations, Des Activités et de La Gestion Administrative de La Commission de Protection Du Territoire Agricole Du Québec. Observations, Conclusions et Recommandations.

Commission de planification de l'agriculture canadienne. 1969. L'agriculture Canadienne Des Années 1970. Ottawa.

Commission de protection du territoire agricole. 2018. http://www. cptaq.gouv.qc.ca/.

Commission Héon. 1955. Rapport Du Comité d'enquête Pour La Protection Des Agriculteurs et Des Consommateurs. Québec.

Commission royale d'enquête sur l'agriculture au Québec. 1967. «L'assainissement Des Sols Au Québec: Rapport de La Commission Royale d'enquête Sur L'agriculture Au Québec.» Québec: La Commission.

Commission sur l'avenir de l'agriculture et de l'agroalimentaire québécois. 2008. Agriculture et Agroalimentaire: Assurer et Bâtir L'avenir. Propositions Pour Une Agriculture Durable et En Santé.

Côté, Michel, Guy Mercier, and Francis Roy. 2014. «L'urbanisation de La Campagne. Motifs et Options Du Régime Québécois de Protection Du Territoire Agricole. » Cahiers de Géographie Du Québec 58 (165): 391-409.

Debailleul, Gary. 1988. «Zonage et Agriculture Québécoise Dans Les Années 1970. » Recherches Sociographiques 29 (2-3): 397-416.

Fortier, Gérard. 1978. «Le Zonage: Excellent Mais Incomplet, La Terre de Chez Nous, 2.

Francoeur, Julie. 2018. «La Place de La Paysannerie Dans L'agriculture et L'espace Rural Québécois: Enjeux Théoriques et d'action Publique. » PhD thesis, Université du Québec à Montréal.

Galeski, Boguslaw. 1967. «Sociologie de La Profession d'agriculteur. » Études Rurales, no. 25/26: 125-39.

Girouard, Benoit (2012) Les «vrais agriculteurs»!, point de vue. Le Soleil, Québec, 25 janvier 2012 [En ligne]. https://www.lesoleil. com/opinions/point-de-vue/les-vrais-agriculteurs-ed1b531bd13229446ef82bef0a8448d4

Gouvernement du Canada, Statistique Canada. 2016. «Recensement de L'agriculture de 2016, » https://www.statcan.gc.ca/fra/ra2016.

Grignon, Claude. 1982. «Professionnalisation et Transformation de La Hiérarchie Sociale Des Agriculteurs, » Économie Rurale 152 (1): 61-66.

Hanna, Kevin S. 1997. "Regulation and Land-Use Conservation: A Case Study of the British Columbia Agricultural Land Reserve." Journal of Soil and Water Conservation 52 (3): 166-70. 
Institut de recherche et de développement en agroenvironnement. 2019. «Inventaire Des Terres Du Canada.» https://www.irda.qc.ca/ $\mathrm{fr} /$ services/protection-ressources/sante-sols/information-sols/inventaire-terres-canada/.

La Terre de chez nous. 1967. «Les Surplus Agricoles: Obstacle Majeur à La Parité Entre Le Secteur Industriel et Le Secteur Agricole. » La Terre de Chez Nous, 10.

Lavoie, Gilles. 1979. «La Stabilisation Des Revenus Agricoles Au Québec et Au Canada. »Économie Rurale 130 (1): 23-26.

Lebeau, Guy. 2015. Notes déposées à la CAPERN suite à la présentation de Guy Lebeau le 19 mai 2015, à 10 heures. Commission de l'Agriculture, des Pêcheries, de l'Énergie et des Ressources Naturelles, Québec, 8 p.

Lefebvre, Henri. 1974. «La Production de L'espace. » L'Homme et La Société 31 (1): 15-32.

Lemoine, J. B. 1957. «Dans La Province de Québec: Seule Une Classe Agricole Organisée Assurera L'avenir de L'agriculture.» La Terre de Chez Nous, 5.

Leray, C. (2008). L'analyse de contenu: De la théorie à la pratique, la méthode Morin-Chartier. PUQ.

Malassis, Louis. 1979. "Economie Agricole, Agro-Alimentaire et Rurale." Économie Rurale 131 (1): 3-10.

Morisset, Michel (2012) Pour une professionnalisation des agriculteurs, point de vue. Le Soleil, Québec, 24 janvier 2012 [En ligne]. http:// www.lapresse.ca/le-soleil/opinions/points-de-vue/201201/23/014488530-pour-une-professionnalisation-des-agriculteurs.php

Morisset, Michel, and Jean-Michel Couture. 2010. Politique et Syndicalisme Agricoles Au Québec. Presses de I'Université Laval.

Mouchet, C., \& Le Clanche, J. F. (2016). Un nouveau regard sur les petites exploitations. Pour, (4), 209-215.

Mundler, Patrick et Fernande Ouellet. 2017. «Qui Est Agriculteur Au Québec? Ambiguïté Des Institutions et Enjeux Pour Le Développement Rural. » Cahiers de Géographie Du Québec, no. 172: 9-32.

Nantel, Lyne (2015). Agriculture: les limites du modèle agricole québécois. Dépossession: une histoire économique du Québec contemporain, 1, 25-68.

Newman, Lenore, Lisa Jordan Powell, and Hannah Wittman. 2015. "Landscapes of Food Production in Agriburbia: Farmland Protection and Local Food Movements in British Columbia." Journal of Rural Studies 39: 99-110.

Organisation de coopération et de développement économiques. 1978. L'agriculture à Temps Partiel: Autriche, France, Belgique, Canada, Finlande, Irlande, Italie, Pays-Bas, Nouvelle-Zélande, Suisse.

-. 2010. Examen de L'OCDE Des Politiques Rurales, Québec, Canada.

Ouimet, Bernard. 2009. Protection Du Territoire Agricole et développement Régional: Une Nouvelle Dynamique Mobilisatrice Pour Nos Communautés: Rapport Remis Au Ministre de L'agriculture, Des Pêcheries et de L'alimentation Du Québec. Ministère de l'agriculture, des pêcheries et de l'alimentation du Québec.

-. 2015. Notes déposées par Bernard Ouimet à la suite de sa présence, le 14 mai 2015, à la Commission de l'agriculture, des pêcheries, de l'énergie et des ressources naturelles. Commission de l'Agriculture, des Pêcheries, de l'Énergie et des Ressources Naturelles, Québec, $9 \mathrm{p}$.
Pierce, J.T., and O.J. Furuseth. 1982. "Farmland Protection Planning in British Columbia." GeoJournal 6 (6): 555-60.

Ploeg, Jean Douwe van der. 2012. The New Peasantries: Struggles for Autonomy and Sustainability in an Era of Empire and Globalization. Routledge.

Pronovost, Jean. 2016. À L'écoute de La Relève Agricole: Le Vécu et Les Attentes Des Jeunes Agriculteurs Québécois.

Proulx, Denise, and Lucie Sauvé. 2007. «Porcheries! La Porciculture Intempestive Au Québec. » Montréal, Éditions Écosociété.

Rémy, J. (1987). La crise de professionnalisation en agriculture: les enjeux de la lutte pour le contrôle du titre d'agriculteur. Sociologie du travail, 415-441.

Saint-Pierre, Michel R. 2009. Une Nouvelle Génération de Programmes de Soutien Financier à L'agriculture: Pour Répondre Aux Besoins Actuels et Soutenir L'entrepreneuriat. Ministère de l'agriculture, des pêcheries et de l'alimentation du Québec.

Sanscartier, R. (2011). Protection du territoire agricole et dynamiques territoriales au Québec: une analyse de la prise en compte des particularités régionales dans le processus décisionnel de la CPTAQ.

Schmitt, Bertrand, and Jean-Louis Coujard. 1985. «Réorientation Des Exploitations et Transformation de L'espace Agricole. » Économie Rurale 166 (1): 54-56.

SOQUIJ. http://citoyens.soquij.qc.ca/ [site consulté le 8 novembre 2016]

Stobbe, Tracy, Alison J. Eagle, and G. Cornelis van Kooten. 2008. "Hobby Farms and Protection of Farmland in British Columbia."

Stobbe, Tracy E., Alison J. Eagle, Geerte Cotteleer, and G. Cornelis van Kooten. 2011. "Farmland Preservation Verdicts-Rezoning Agricultural Land in British Columbia." Canadian Journal of Agricultural Economics/Revue Canadienne d'agroeconomie 59 (4): 555-72.

Union des producteurs agricoles. 2015. Pour nourrir le Québec: Protégeons nos terres, priorisons l'agriculture. Commission de l'Agriculture, des Pêcheries, de l'Énergie et des Ressources Naturelles, Québec, $34 \mathrm{p}$.

—. 2018. «La Trousse d'information PDZA. » http://trousse.pdza.ca/. Vachon, Bernard. 2011. «La Passion Du Rural. » Quarante Ans d'écrits, de Paroles et d'actions Pour Que Vive Le Québec Rural 1. 